\title{
Correction: Predictors of fluoroquinolone-resistant bacteria in the rectal vault of men undergoing prostate biopsy
}

Wei Phin Tan $\circledast^{1} \cdot$ Dimitri Papagiannopoulos ${ }^{1} \cdot$ Kalyan C. Latchamsetty ${ }^{1} \cdot$ Nathaniel Wilson $^{1} \cdot$ Nicholas $^{\prime}$ Block $^{1} \cdot$ Lester Raff ${ }^{2} \cdot$ Alfredo Mena Lora $^{3} \cdot$ Christopher L. Coogan $^{1,2} \cdot$ Michael R. Abern $^{4}$

Published online: 31 January 2019

(C) Springer Nature America, Inc. 2019

Correction to: Prostate Cancer and Prostatic Diseases; https://doi.org/10.1038/s41391-018-0092-3; published online 02 October 2018.
The original version of this article contained an error in the name of author Alfredo Mena Lora. This has now been corrected.

Wei Phin Tan

tanweiphin@yahoo.com

1 Division of Urology, Rush University Medical Center,

Chicago, IL, USA

2 Uropartners LLC, Chicago, IL, USA

3 Division of Infectious Diseases, Department of Medicine,

University of Illinois Chicago, Chicago, IL, USA

4 Department of Urology, University of Illinois Chicago,

Chicago, IL, USA 